\title{
Increased serum asprosin is correlated with diabetic nephropathy
}

\author{
Rui Wang ${ }^{1 \dagger}$, Peng Lin ${ }^{2 \dagger}$, Huibo Sun ${ }^{3 *}$ and Wenchao $\mathrm{Hu}^{3 *}$
}

\begin{abstract}
Objective: The adipokine asprosin, which was recently discovered, facilitates hepatic glucose production. The aim of this study is to see whether serum asprosin concentrations are linked to diabetic nephropathy (DN).

Methods: We performed this investigation in a group of 212 type 2 diabetes (T2DM) patients. These patients were classified into three subgroups: DN0 group (normal to mildly increased), DN1 group (moderately increased), and DN2 group (severely increased) on the basis of urine albumin-to-creatinine ratio (ACR).

Results: When compared to the controls, T2DM patients had higher serum asprosin levels. The DN2 group had significantly higher serum asprosin than the DNO and DN1 groups. Furthermore, the DN1 group had higher serum asprosin than the DNO group. Serum asprosin was linked to a higher risk of T2DM and DN in a logistic regression analysis. Serum asprosin was found to be positively related with disease duration, systolic blood pressure, blood urea nitrogen, creatinine, uric acid, ACR, calcium channel blockers, and angiotensin-converting enzyme inhibitor/angiotensin II receptor blocker therapy, but negatively related with glomerular filtration rate, metformin, and acarbose therapy.
\end{abstract}

Conclusion: Serum asprosin increase with the progression of DN. Serum asprosin is correlated with renal function and ACR.

Keywords: Asprosin, Diabetic nephropathy, Renal function, Urine albumin to creatinine ratio

\section{Introduction}

Diabetes mellitus is becoming more common around the world [1]. Diabetic nephropathy (DN), the most frequent diabetic complication, primarily caused the result of end-stage renal disease (ESRD) [2]. DN is responsible for 25-35 percent of type 2 diabetes mellitus (T2DM) cases and significantly raises T2DM patient mortality [3]. The underlying mechanism of DN is currently being investigated. However, the precise mechanism of DN is still uncertain [4]. Serum biomarkers, such as glycated albumin, copeptin, cystatin C, 8-hydroxy-2'-deoxyguanine

\footnotetext{
*Correspondence: 981110887@qq.com; tjmuhwc@126.com

${ }^{\dagger}$ Rui Wang and Peng Lin contributed equally to this article

${ }^{3}$ Department of Endocrinology, Qilu Hospital (Qingdao), Cheeloo

College of Medicine, Shandong University, 758 Hefei Road, Shibei District, Qingdao 266035, Shandong, China
}

Full list of author information is available at the end of the article have been recently utilized to diagnose $\mathrm{DN}$ or assess the progression of DN [5-8]. Investigating new predictive biomarkers will provide an opportunity for preventive or therapeutic interventions to prevent or delay the occurrence of ESRD.

Asprosin, a C-terminal cleavage peptide developed by profibrillin [9], is a recently found adipokine. Asprosin is released from white adipose and transferred to the liver. Asprosin causes hepatic gluconeogenesis and results in increased circulating insulin and glucose concentrations [9]. Plasma asprosin permeates the blood-brain barrier and stimulates orexigenic neurons directly through a cAMP-dependent signal, triggering appetite stimulation and weight gain [10]. In humans, a genetic mutation of asprosin causes a condition characterized by a lack of appetite and excessive leanness [10].

Recent research has found a relation between serum asprosin and diabetes. Serum asprosin are substantially 
higher in T2DM subjects than in controls [11-13]. Furthermore, plasma asprosin levels are substantially higher in the impaired glucose tolerance compared with normal controls [14]. Criculating asprosin has also been demonstrated to be related with fasting blood glucose and glycosylated hemoglobin [12, 14]. However, no prior research has looked into the relation between asprosin and diabetic complications. As a result, we expect to test the hypothesis that serum asprosin levels are linked to DN.

\section{Materials and methods \\ Patients}

This investigation was conducted in 212 T2DM patients who were enrolled from the inpatient Department of Endocrinology of Qilu Hospital. These subjects were then classified into three groups based on the measurements of the urinary albumin to creatinine ratio (ACR) [15]: DN0 (normal to mildly increased, ACR $<30 \mathrm{mg} / \mathrm{g}, 94$ subjects), DN1 (moderately increased, $30 \leq \mathrm{ACR} \leq 300 \mathrm{mg} / \mathrm{g}, 82$ subjects), and DN2 (severely increased, ACR $>300 \mathrm{mg} / \mathrm{g}$, 36 subjects).

The following were the exclusion criteria: type 1 diabetes, cancer, and acute infection during the preceding 3 months. The control group consisted of 72 healthy adults. They performed an oral glucose tolerance test and found that it was normal.

The hospital ethics board approved this report, and it was performed in accordance with the Declaration of Helsinki.

\section{Measurements}

Anthropometric information including height, weight, blood pressure, and therapy information such as antihypertensive drugs, statins, and anti-diabetic drugs were recorded. The body mass index (BMI) was determined a formula as weight/height ${ }^{2}$. The Cockroft-Gault formula was used to measure the glomerular filtration rate (GFR).

A biochemistry automated analyzer (Hitachi 7170, Tokyo, Japan) was used to measure blood lipids, renal function parameters. High-performance liquid chromatography was used to evaluate HbA1c levels. The ACR was assessed three times and the average results were used. A commercial ELISA kit was used to test serum samples for asprosin (Phoenix Pharmaceuticals, Inc, USA). The results were checked in duplicate.

\section{Statistical analysis}

The data is viewed as the forms of means \pm standard deviations or median (interquartile). ANOVA, Chi-square, and Kruskal-Wallis test were used to assess variable differences between groups. Logistic regression analysis was conducted to evaluate the variables related with T2DM and DN. Linear regression analysis was used to evaluate the relationship between serum asprosin and other variables. $P<0.05$ was set as statistical significant.

\section{Results \\ Baseline parameter comparison}

Systolic blood pressure (SBP), HbA1c were higher, while high-density lipoprotein cholesterol (HDL-C) was lower in the case group compared to controls (Table 1). The DN2 group also had substantially higher SBP, blood urea nitrogen (BUN), creatinine (Cr), and uric acid (UA) as well as lower GFR compared to DN1, DN0, and control groups (Table 1).

As displayed in Table 1, the DN2 and DN1 subjects had longer disease duration, blood pressure, higher proportion of angiotensin-converting enzyme inhibitor (ACEI)/ angiotensin II receptor blockers (ARB) and calcium channel blockers (CCB) therapy than DN0 subjects. Furthermore, the DN2 subjects had longer disease duration and a higher proportion of CCB and ACEI/ARB therapy than the DN1 subjects.

\section{Serum asprosin differences}

Table 1 shows that when compared to the control group, all T2DM patients had substantially higher serum asprosin. When compared to the DN1 and DN0 subjects, the DN2 subjects had substantially higher serum asprosin. The DN1 subjects also had substantially higher serum asprosin than the DNO subjects.

\section{Relation between serum asprosin and T2DM}

T2DM group had substantially higher serum asprosin than the control group $[17.06(13.92-19.57) \mathrm{ng} / \mathrm{mL}$ vs. $11.86(9.17-14.56) \mathrm{ng} / \mathrm{mL}, P<0.001]$. Table 2 shows that SBP, diastolic blood pressure (DBP), HDL-C, BUN, and serum asprosin were all related to T2DM in a univariate logistic regression model. Serum asprosin was still related to T2DM after adjusting the confounding factors.

\section{Relation between serum asprosin and DN}

T2DM groups with moderately and severely increased ACR were considered as the DN group. DN group had substantially higher asprosin than non-DN group [18.3 (15.01-20.55) $\mathrm{ng} / \mathrm{mL}$ vs. $15.43(12.95-18.26) \mathrm{ng} / \mathrm{mL}$, $P<0.001]$. Table 3 shows disease duration, SBP, DBP, lowdensity lipoprotein cholesterol (LDL-C), Total cholesterol (TC), BUN, Cr, GFR, serum asprosin, CCB, ACEI/ $A R B$ therapy were all related to DN in a univariate logistic regression model. Serum asprosin was still related to DN after adjusting the confounding factors (Table 3).

\section{Correlation of serum asprosin with other variables}

After a simple linear regression study, serum asprosin was related to disease duration, SBP, ACR, BUN, Cr, UA, 
Table 1 Clinical characteristic of T2DM patients and controls

\begin{tabular}{|c|c|c|c|c|c|}
\hline & \multirow[t]{2}{*}{ Control } & \multicolumn{4}{|l|}{ T2DM patients } \\
\hline & & DNO & DN1 & DN2 & $p$ \\
\hline$N$ & 72 & 94 & 82 & 36 & \\
\hline Age (years) & $58.42 \pm 8.01$ & $57.55 \pm 11.57$ & $58.39 \pm 13.22$ & $59.78 \pm 11.32$ & 0.791 \\
\hline Gender (M/F) & $41 / 31$ & $51 / 43$ & $41 / 41$ & $19 / 17$ & 0.855 \\
\hline Duration (years) & - & $8.53 \pm 1.68$ & $10.15 \pm 2.43^{b}$ & $12.06 \pm 2.34^{b c}$ & $<0.001$ \\
\hline $\mathrm{BMI}\left(\mathrm{kg} / \mathrm{m}^{2}\right)$ & $25.88 \pm 2.85$ & $26.25 \pm 4.05$ & $26.16 \pm 3.5$ & $26.32 \pm 3.1$ & 0.901 \\
\hline $\mathrm{SBP}(\mathrm{mmHg})$ & $122.01 \pm 11.48$ & $135.8 \pm 22.39^{a}$ & $146.71 \pm 30.57^{\mathrm{ab}}$ & $156.69 \pm 22.51^{a b c}$ & $<0.001$ \\
\hline $\mathrm{DBP}(\mathrm{mmHg})$ & $78.96 \pm 7.89$ & $80.85 \pm 14.75$ & $87.56 \pm 20.22^{a b}$ & $85.69 \pm 12.83^{a b}$ & 0.002 \\
\hline HbA1c (\%) & $4.75 \pm 0.31$ & $7.82 \pm 1.48^{\mathrm{a}}$ & $8.1 \pm 1.21^{\mathrm{a}}$ & $7.76 \pm 1.48^{\mathrm{a}}$ & $<0.001$ \\
\hline $\mathrm{TG}(\mathrm{mmol} / \mathrm{L})$ & $2.04 \pm 1.59$ & $1.88 \pm 1.14$ & $2.04 \pm 1.65$ & $2.13 \pm 1.12$ & 0.775 \\
\hline $\mathrm{TC}(\mathrm{mmol} / \mathrm{L})$ & $5.28 \pm 0.91$ & $5 \pm 0.98$ & $5.41 \pm 1.2$ & $5.42 \pm 1.02^{b}$ & 0.041 \\
\hline $\mathrm{HDL}-\mathrm{C}(\mathrm{mmol} / \mathrm{L})$ & $1.5 \pm 0.24$ & $1.11 \pm 0.23^{\mathrm{a}}$ & $1.13 \pm 0.21^{\mathrm{a}}$ & $1.16 \pm 0.33^{\mathrm{a}}$ & $<0.001$ \\
\hline LDL-C (mmol/L) & $3.28 \pm 0.54$ & $3.33 \pm 0.81$ & $3.65 \pm 0.99^{a b}$ & $3.61 \pm 0.8^{\mathrm{a}}$ & 0.008 \\
\hline BUN (mmol/L) & $5.42 \pm 1.18$ & $5.36 \pm 1.64$ & $5.9 \pm 1.97$ & $8.85 \pm 4.1^{\mathrm{abc}}$ & $<0.001$ \\
\hline $\mathrm{Cr}(\mu \mathrm{mol} / \mathrm{L})$ & $66.6 \pm 10.65$ & $66.32 \pm 18.91$ & $65.95 \pm 21.12$ & $120.75 \pm 72.65^{a b c}$ & $<0.001$ \\
\hline $\mathrm{UA}(\mu \mathrm{mol} / \mathrm{L})$ & $305.11 \pm 70.95$ & $301.42 \pm 87.54$ & $296.39 \pm 76.9$ & $356.22 \pm 88.31^{a b c}$ & 0.002 \\
\hline $\mathrm{ACR}(\mathrm{mg} / \mathrm{g})$ & - & $16.14 \pm 4.48$ & $96.08 \pm 83.67^{b}$ & $>300^{\mathrm{bc}}$ & $<0.001$ \\
\hline GFR (mL/min/1.73 m²) & $101.66 \pm 12.09$ & $109.26 \pm 41.65$ & $109.14 \pm 33.24$ & $67.46 \pm 35.53^{\mathrm{abc}}$ & $<0.001$ \\
\hline Asprosin (ng/mL) & $11.86(9.17-14.56)$ & $15.43(12.95-18.26)^{\mathrm{a}}$ & $17.38(13.86-19.56)^{\mathrm{ab}}$ & $22.52(20.25-24.58)^{\mathrm{abc}}$ & $<0.001$ \\
\hline \multicolumn{6}{|l|}{ Treatment } \\
\hline Metformin (n, \%) & - & $68(72.3 \%)$ & $58(70.7 \%)$ & $19(52.8 \%)$ & 0.084 \\
\hline Acarbose $(n, \%)$ & - & $46(48.9 \%)$ & $44(53.7 \%)$ & $13(36.1 \%)$ & 0.213 \\
\hline Sulfonylureas (n, \%) & - & $60(63.8 \%)$ & $52(63.4 \%)$ & $21(58.3 \%)$ & 0.834 \\
\hline DPP-IV inhibitor (n, \%) & & $46(48.9 \%)$ & $36(43.9 \%)$ & $15(41.7 \%)$ & 0.691 \\
\hline Insulin (n, \%) & - & $37(39.4 \%)$ & $26(31.7 \%)$ & $17(47.2 \%)$ & 0.252 \\
\hline Statin $(n, \%)$ & - & $47(50 \%)$ & $45(54.9 \%)$ & $21(58.3 \%)$ & 0.651 \\
\hline $\operatorname{CCB}(n, \%)$ & - & $51(54.3 \%)$ & $59(72 \%)^{b}$ & $31(86.1 \%)^{b c}$ & 0.001 \\
\hline ACEl/ARB (n, \%) & - & 45 (47.9\%) & $55(67.1 \%)^{b}$ & $32(88.9 \%)^{b c}$ & $<0.001$ \\
\hline
\end{tabular}

T2DM type 2 diabetes mellitus, $B M I$ body mass index, SBP systolic blood pressure, DBP diastolic blood pressure, $T G$ triglycerides, $T C$ total cholesterol, $H D L-C$ highdensity lipoprotein cholesterol, $L D L-C$ low-density lipoprotein cholesterol, $B U N$ blood urea nitrogen, $C r$ creatinine, $U A$ uric acid, $A C R$ urine albumin to creatinine ratio, GFR glomerular filtration rate, DPP-IV inhibitor dipeptidyl peptidase IV inhibitor, CCB calcium channel blockers, $A C E I$ angiotensin-converting enzyme inhibitor, $A R B$ angiotensin II receptor blockers

${ }^{\text {a }}$ Significant versus control subjects

b Significant versus DNO group

'Significant versus DN1 group

GFR, metformin, acarbose, CCB, and ACEI/ARB therapy (Table 4). After multiple linear regression analysis, BUN and ACR remained to be related to serum asprosin.

\section{Discussion}

Asprosin, a recently found adipokine, increase circulating glucose and insulin levels by promoting hepatocyte glucose production and output [9]. Lee et al. reported that asprosin incubation promotes the apoptosis and dysfunction of insulinoma cells and human islets through a toll-like receptor 4 (TLR4) /JNK-mediated pathway [16], suggesting that asprosin causes $\beta$ cell dysfunction. Furthermore, asprosin exacerbates insulin sensitivity which is assessed using glucose utilization, and insulin receptor substrate 1 and Akt phosphorylation levels [17]. The asprosin receptor OLFR734 modulates hepatic glucose output [18]. OLFR734 ${ }^{-/-}$mice exhibits reduced hepatic glucose output and increased insulin sensitivity [18]. Asprosin and its receptor OLFR734 might be used as a new mechanism for the T2DM therapy.

Recent study reported that asprosin levels increase at $24 \mathrm{~h}$ after coronary angiography compared with those at the time of admission because of unstable angina pectoris [19]. Changes in asprosin levels are positively correlated with syntax scores which are utilized to assess disease severity [19]. The focus of this study was on 
Table 2 Logistic regression analysis for determining the factors associated with T2DM

\begin{tabular}{|c|c|c|c|c|}
\hline \multirow[t]{2}{*}{ Characteristics } & \multicolumn{2}{|c|}{ Univariate logistic regression } & \multicolumn{2}{|c|}{ Multiple logistic regression } \\
\hline & OR $(95 \% \mathrm{Cl})$ & $P$ & OR $(95 \% \mathrm{Cl})$ & $P$ \\
\hline Age (years) & $0.999(0.975-1.023)$ & 0.916 & - & - \\
\hline Gender (M/F) & $1.203(0.702-2.063)$ & 0.501 & - & - \\
\hline BMI $\left(\mathrm{kg} / \mathrm{m}^{2}\right)$ & $1.029(0.952-1.113)$ & 0.47 & - & - \\
\hline $\mathrm{SBP}(\mathrm{mmHg})$ & $1.055(1.036-1.075)$ & $<0.001$ & $1.112(1.06-1.168)$ & $<0.001$ \\
\hline $\mathrm{DBP}(\mathrm{mmHg})$ & $1.029(1.006-1.051)$ & 0.012 & $1.021(1.002-1.043)$ & 0.016 \\
\hline TG $(\mathrm{mmol} / \mathrm{L})$ & $0.974(0.811-1.171)$ & 0.782 & - & - \\
\hline $\mathrm{TC}(\mathrm{mmol} / \mathrm{L})$ & $0.955(0.741-1.231)$ & 0.723 & - & - \\
\hline $\mathrm{HDL}-\mathrm{C}(\mathrm{mmol} / \mathrm{L})$ & $0.004(0.001-0.016)$ & $<0.001$ & $0.002(0.001-0.017)$ & $<0.001$ \\
\hline LDL-C (mmol/L) & $1.415(0.996-2.009)$ & 0.052 & - & - \\
\hline BUN (nmol/L) & $1.189(1.023-1.382)$ & 0.024 & $1.087(0.821-1.441)$ & 0.56 \\
\hline $\mathrm{Cr}(\mu \mathrm{mol} / \mathrm{L})$ & $1.011(0.999-1.023)$ & 0.08 & - & - \\
\hline $\mathrm{UA}(\mu \mathrm{mol} / \mathrm{L})$ & $1.001(0.997-1.004)$ & 0.743 & - & - \\
\hline $\mathrm{GFR}\left(\mathrm{mL} / \mathrm{min} / 1.73 \mathrm{~m}^{2}\right)$ & $1(0.993-1.008)$ & 0.924 & - & - \\
\hline Asprosin (ng/mL) & $1.623(1.428-1.843)$ & $<0.001$ & $1.635(1.373-1.947)$ & $<0.001$ \\
\hline
\end{tabular}

Abbreviation as Table 1

Table 3 Logistic regression analysis for determining the factors associated with DN

\begin{tabular}{|c|c|c|c|c|}
\hline \multirow[t]{2}{*}{ Characteristics } & \multicolumn{2}{|c|}{ Univariate logistic regression } & \multicolumn{2}{|c|}{ Multiple logistic regression } \\
\hline & OR $(95 \% \mathrm{Cl})$ & $P$ & OR $(95 \% \mathrm{Cl})$ & $P$ \\
\hline Age (years) & $1.009(0.986-1.031)$ & 0.453 & - & - \\
\hline Gender (M/F) & $1.147(0.666-1.973)$ & 0.622 & - & - \\
\hline Duration (years) & $1.672(1.404-1.991)$ & $<0.001$ & $1.599(1.318-1.941)$ & $<0.001$ \\
\hline $\mathrm{BMI}\left(\mathrm{kg} / \mathrm{m}^{2}\right)$ & $0.997(0.926-1.074)$ & 0.94 & - & - \\
\hline $\mathrm{SBP}(\mathrm{mmHg})$ & $1.022(1.01-1.034)$ & $<0.001$ & $1.012(0.988-1.036)$ & 0.332 \\
\hline $\mathrm{DBP}(\mathrm{mmHg})$ & $1.024(1.006-1.043)$ & 0.011 & $1.008(0.98-1.038)$ & 0.572 \\
\hline $\mathrm{HbA1c}(\%)$ & $1.094(0.898-1.333)$ & 0.372 & - & - \\
\hline $\mathrm{TG}(\mathrm{mmol} / \mathrm{L})$ & $1.116(0.9-1.384)$ & 0.316 & - & - \\
\hline $\mathrm{TC}(\mathrm{mmol} / \mathrm{L})$ & $1.451(1.104-1.907)$ & 0.008 & $1.845(0.581-5.861)$ & 0.299 \\
\hline $\mathrm{HDL}-\mathrm{C}(\mathrm{mmol} / \mathrm{L})$ & $1.782(0.575-5.519)$ & 0.317 & - & - \\
\hline LDL-C (mmol/L) & $1.525(1.098-2.118)$ & 0.012 & $0.659(0.163-2.668)$ & 0.559 \\
\hline BUN (nmol/L) & $1.326(1.142-1.539)$ & $<0.001$ & $1.301(1.02-1.661)$ & 0.034 \\
\hline $\mathrm{Cr}(\mu \mathrm{mol} / \mathrm{L})$ & $1.015(1.004-1.026)$ & 0.008 & $0.995(0.974-1.016)$ & 0.639 \\
\hline $\mathrm{UA}(\mu \mathrm{mol} / \mathrm{L})$ & 1.002 (0.999-1.005) & 0.267 & - & - \\
\hline $\mathrm{GFR}\left(\mathrm{mL} / \mathrm{min} / 1.73 \mathrm{~m}^{2}\right)$ & $0.991(0.984-0.999)$ & 0.028 & $1.005(0.993-1.017)$ & 0.397 \\
\hline Asprosin (ng/mL) & $1.242(1.142-1.35)$ & $<0.001$ & $1.183(1.064-1.315)$ & 0.002 \\
\hline \multicolumn{5}{|l|}{ Treatment } \\
\hline Metformin $(n, \%)$ & $0.718(0.398-1.295)$ & 0.271 & - & - \\
\hline Acarbose (n, \%) & $0.975(0.567-1.677)$ & 0.927 & - & - \\
\hline Sulfonylureas (n, \%) & $0.919(0.524-1.611)$ & 0.769 & - & - \\
\hline DPP-IV inhibitor $(\mathrm{n}, \%)$ & $0.794(0.461-1.369)$ & 0.407 & - & - \\
\hline Insulin (n, \%) & $0.883(0.505-1.544)$ & 0.663 & - & - \\
\hline Statin $(n, \%)$ & $1.269(0.737-2.186)$ & 0.39 & - & - \\
\hline CCB $(n, \%)$ & $2.71(1.507-4.874)$ & 0.001 & $1.023(0.382-2.736)$ & 0.964 \\
\hline $\operatorname{ACEI} / \operatorname{ARB}(n, \%)$ & $3.056(1.718-5.436)$ & $<0.001$ & $1.348(0.508-3.573)$ & 0.549 \\
\hline
\end{tabular}

Abbreviation as Table 1 
Table 4 The correlation between serum asprosin concentrations and various parameters in T2DM patients

\begin{tabular}{|c|c|c|c|c|}
\hline \multirow[t]{2}{*}{ Parameters } & \multicolumn{2}{|c|}{$\begin{array}{l}\text { Simple regression } \\
\text { analysis }\end{array}$} & \multicolumn{2}{|c|}{$\begin{array}{l}\text { Multiple regression } \\
\text { analysis }\end{array}$} \\
\hline & $\mathbf{R}$ & $P$ & $\beta$ & $P$ \\
\hline Age (years) & 0.066 & 0.338 & & \\
\hline Gender (M/F) & 0.015 & 0.824 & & \\
\hline Duration (years) & 0.235 & 0.001 & 0.023 & 0.746 \\
\hline $\mathrm{BMI}\left(\mathrm{Kg} / \mathrm{m}^{2}\right)$ & 0.034 & 0.619 & & \\
\hline SBP $(m m H g)$ & 0.22 & 0.001 & 0.087 & 0.302 \\
\hline $\mathrm{DBP}(\mathrm{mmHg})$ & 0.07 & 0.31 & & \\
\hline HbA1c (\%) & 0.065 & 0.35 & & \\
\hline TG $(m m o l / L)$ & 0.05 & 0.472 & & \\
\hline $\mathrm{TC}(\mathrm{mmol} / \mathrm{L})$ & 0.043 & 0.533 & & \\
\hline $\mathrm{HDL}-\mathrm{C}(\mathrm{mmol} / \mathrm{L})$ & -0.007 & 0.917 & & \\
\hline LDL-C (mmol/L) & 0.032 & 0.644 & & \\
\hline BUN (nmol/L) & 0.404 & $<0.001$ & 0.219 & 0.03 \\
\hline $\mathrm{Cr}(\mu \mathrm{mol} / \mathrm{L})$ & 0.304 & $<0.001$ & 0.159 & 0.158 \\
\hline UA ( $\mu \mathrm{mol} / \mathrm{L})$ & 0.163 & 0.018 & 0.09 & 0.21 \\
\hline ACR (mg/g) & 0.466 & $<0.001$ & 0.323 & $<0.001$ \\
\hline GFR (mL/min/1.73 m²) & -0.283 & $<0.001$ & 0.078 & 0.356 \\
\hline \multicolumn{5}{|l|}{ Treatment } \\
\hline Metformin (n, \%) & -0.143 & 0.037 & -0.009 & 0.907 \\
\hline Acarbose (n, \%) & -0.148 & 0.031 & -0.08 & 0.282 \\
\hline Sulfonylureas (n, \%) & -0.121 & 0.078 & & \\
\hline DPP-IV inhibitor (n, \%) & -0.072 & 0.295 & & \\
\hline Insulin (n, \%) & 0.079 & 0.255 & & \\
\hline Statin (n, \%) & 0.009 & 0.897 & & \\
\hline CCB $(n, \%)$ & 0.163 & 0.018 & -0.002 & 0.997 \\
\hline ACEI/ARB $(n, \%)$ & 0.23 & 0.001 & 0.069 & 0.423 \\
\hline
\end{tabular}

Abbreviation as Table 1

macrovascular disease. Asprosin can be related to diabetic macrovascular complications according to this report. Our findings revealed a relation between elevated serum asprosin concentrations and DN. Another investigation showed that serum asprosin is independently associated with ACR in T2DM population [20]. Circulating asprosin may be used as a novel serum biomarker for DN evaluation.

Asprosin incubation promotes inflammation reaction via the TLR4/JNK-mediated signal pathway in insulinoma cells and human islets [13]. Asprosin treatment also induces inflammation markers including NF- $\kappa \beta$, interleukin-6, and phosphorylated ІкB [14]. These findings revealed a strong relation between asprosin and inflammation. Low-grade inflammation is characterized as an innate immune system reaction that has been activated [21]. Increased circulating pro-inflammatory cytokines that stimulate the immune system are clinically identified as this form of inflammation. Chronic inflammation is related to the mechanism of
T2DM and DN [22]. As a result, asprosin is thought to play a role in $\mathrm{DN}$ through regulating inflammatory response.

There are a few limitations in this report. First, the claim is challenged by the small sample size. Second, the data's cross-sectional design limits the conclusion's intensity. Future longitudinal research would be required to validate the causal relationship. Last, the healthy controls were recruited from the subjects for health check-up. They all did OGTT to exclude T2DM upon their own request. Therefore, they may have some risk factors for diabetes or worry about developing diabetes. This possibility might have introduced bias to the final results.

\section{Conclusions}

In conclusion, as the disease progresses, serum asprosin rise with the progression of DN. Serum asprosin is related to renal function and ACR. In addition to conventional approaches for assessing the risk and progression of $\mathrm{DN}$, serum asprosin may be used as a potential serum biomarker.

Acknowledgments

No acknowledgments.

Authors' contributions

HS and WH planed this study. RW and PL collected the data, performed the ELISA measurement. WH did the statistical work. All authors read and approved the final manuscript.

\section{Funding}

Funded by the National Natural Science Foundation of China (82000737), Qingdao Outstanding Health Professional Development Fund, and the

National Natural Science Foundation of China (82070799).

Availability of data and materials

Data are available upon reasonable request.

\section{Declarations}

Ethics approval and consent to participate

The hospital ethics board of Qilu Hospital approved this report. Informed consent was obtained from all participants.

\section{Consent for publication}

All authors approved the paper publication.

\section{Competing interests}

The authors declare no conflict of interest.

\section{Author details}

'Department of Blood Transfusion, Qilu Hospital (Qingdao), Cheeloo College of Medicine, Shandong University, Qingdao, People's Republic of China.

${ }^{2}$ Department of Endocrinology, Qilu Hospital, Cheeloo College of Medicine, Shandong University, Jinan, People's Republic of China. ${ }^{3}$ Department of Endocrinology, Qilu Hospital (Qingdao), Cheeloo College of Medicine, Shandong University, 758 Hefei Road, Shibei District, Qingdao 266035, Shandong, China.

Received: 10 January 2021 Accepted: 19 April 2021

Published online: 01 May 2021 


\section{References}

1. Jansson SP, Fall K, Brus O, Magnuson A, Wändell P, Östgren CJ, et al. Prevalence and incidence of diabetes mellitus: a nationwide populationbased pharmaco-epidemiological study in Sweden. Diabet Med. 2015:32:1319-28.

2. Kakio Y, Uchida HA, Takeuchi H, Okuyama Y, Okuyama M, Umebayashi $R$, et al. Diabetic nephropathy is associated with frailty in patients with chronic hemodialysis. Geriatr Gerontol Int. 2018;18:1597-602.

3. Eppens MC, Craig ME, Cusumano J, Hing S, Chan AK, Howard NJ, et al. Prevalence of diabetes complications in adolescents with type 2 compared with type 1 diabetes. Diabetes Care. 2006;29:1300-6.

4. Gallagher H, Suckling RJ. Diabetic nephropathy: where are we on the journey from pathophysiology to treatment? Diabetes Obes Metab. 2016;18:641-7.

5. Bellia C, Cosma C, Lo Sasso B, Bivona G, Agnello L, Zaninotto M, et al. Glycated albumin as a glycaemic marker in patients with advanced chronic kidney disease and anaemia: a preliminary report. Scand J Clin Lab Invest. 2019:79:293-7.

6. Bellia C, Zaninotto M, Cosma C, Agnello L, Bivona G, Marinova M, et al. Clinical usefulness of glycated albumin in the diagnosis of diabetes: results from an Italian study. Clin Biochem. 2018;54:68-72.

7. Papadopoulou-Marketou N, Kanaka-Gantenbein C, Marketos N, Chrousos GP, Papassotiriou I. Biomarkers of diabetic nephropathy: a 2017 update. Crit Rev Clin Lab Sci. 2017;54:326-42.

8. Huang H, Li D, Huang X, Wang Y, Wang S, Wang X, et al. Association of complement and inflammatory biomarkers with diabetic nephropathy. Ann Clin Lab Sci. 2019:49:488-95.

9. Romere C, Duerrschmid C, Bournat J, Constable P, Jain M, Xia F, et al. Asprosin, a fasting-induced glucogenic protein hormone. Cell. 2016;165:566-79.

10. Duerrschmid C, He Y, Wang C, Li C, Bournat JC, Romere C, et al. Asprosin is a centrally acting orexigenic hormone. Nat Med. 2017;23:1444-53.

11. Zhang L, Chen C, Zhou N, Fu Y, Cheng X. Circulating asprosin concentrations are increased in type 2 diabetes mellitus and independently associated with fasting glucose and triglyceride. Clin Chim Acta. 2019:489:183-8.

12. Li X, Liao M, Shen $R$, Zhang $L, H u H$, Wu J, et al. Plasma asprosin levels are associated with glucose metabolism, lipid, and sex hormone profiles in females with metabolic-related diseases. Mediat Inflamm. 2018;2018:7375294

13. Zhang $X$, Jiang $H$, Ma $X$, Wu H. Increased serum level and impaired response to glucose fluctuation of asprosin is associated with type 2 diabetes mellitus. J Diabetes Investig. 2020;11:349-55.

14. Wang Y, Qu H, Xiong X, Qiu Y, Liao Y, Chen Y, et al. Plasma asprosin concentrations are increased in individuals with glucose dysregulation and correlated with insulin resistance and first-phase insulin secretion. Mediat Inflamm. 2018;2018:9471583.

15. American Diabetes Association. 11. Microvascular complications and foot care: standards of medical care in diabetes-2021. Diabetes Care. 2021:44:S151-67.

16. Lee T, Yun S, Jeong JH, Jung TW. Asprosin impairs insulin secretion in response to glucose and viability through TLR4/JNK-mediated inflammation. Mol Cell Endocrinol. 2019;486:96-104.

17. Jung TW, Kim HC, Kim HU, ParkT, Park J, Kim U, et al. Asprosin attenuates insulin signaling pathway through PKC $\delta$-activated ER stress and inflammation in skeletal muscle. J Cell Physiol. 2019;234:20888-99.

18. Li E, Shan H, Chen L, Long A, Zhang Y, Liu Y, et al. OLFR734 mediates glucose metabolism as a receptor of asprosin. Cell Metab. 2019;30:319-28.

19. Acara AC, Bolatkale M, Kızıloğlu I, İbişoğlu E, Can Ç. A novel biochemical marker for predicting the severity of ACS with unstable angina pectoris: asprosin. Am J Emerg Med. 2018;36:1504-5.

20. Deng X, Zhao L, Guo C, Yang L, Wang D, Li Y, et al. Higher serum asprosin level is associated with urinary albumin excretion and renal function in type 2 diabetes. Diabetes Metab Syndr Obes. 2020;13:4341-51.

21. Ahechu P, Zozaya G, Martí P, Hernández-Lizoáin JL, Baixauli J, Unamuno X et al. NLRP3 inflammasome: a possible link between obesity-associated low-grade chronic inflammation and colorectal cancer development. Front Immunol. 2018;9:2918.

22. Matoba K, Takeda Y, Nagai Y, Kawanami D, Utsunomiya K, Nishimura R. Unraveling the role of inflammation in the pathogenesis of diabetic kidney disease. Int J Mol Sci. 2019;20:3393.

\section{Publisher's Note}

Springer Nature remains neutral with regard to jurisdictional claims in published maps and institutional affiliations.
Ready to submit your research? Choose BMC and benefit from:

- fast, convenient online submission

- thorough peer review by experienced researchers in your field

- rapid publication on acceptance

- support for research data, including large and complex data types

- gold Open Access which fosters wider collaboration and increased citations

- maximum visibility for your research: over 100M website views per year

At $B M C$, research is always in progress.

Learn more biomedcentral.com/submissions 